\title{
Role of Electron-Hole Pair Excitations in the Dissociative Adsorption of Diatomic Molecules on Metal Surfaces
}

\author{
J. I. Juaristi, ${ }^{1,2,3}$ M. Alducin, ${ }^{2,3}$ R. Díez Muiño, ${ }^{2,3}$ H. F. Busnengo, ${ }^{4}$ and A. Salin ${ }^{3}$ \\ ${ }^{1}$ Departamento de Física de Materiales, Facultad de Químicas, UPV/EHU, Apartado 1072, 20080 San Sebastián, Spain \\ ${ }^{2}$ Centro de Física de Materiales Centro Mixto CSIC-UPV/EHU, Edificio Korta, Avenida de Tolosa 72, 20018 San Sebastián, Spain \\ ${ }^{3}$ Donostia International Physics Center DIPC, P. Manuel de Lardizabal 4, 20018 San Sebastián, Spain \\ ${ }^{4}$ Instituto de Física Rosario (CONICET-UNR) and Facultad de Ciencias Exactas, Ingeniería y Agrimensura, \\ Universidad Nacional de Rosario, Avenida Pellegrini 250, 2000 Rosario, Argentina
}

(Received 15 November 2007; published 20 March 2008)

\begin{abstract}
We quantitatively evaluate the contribution of electron-hole pair excitations to the reactive dynamics of $\mathrm{H}_{2}$ on $\mathrm{Cu}(110)$ and $\mathrm{N}_{2}$ on $\mathrm{W}(110)$, including the six dimensionality of the process in the entire calculation. The interaction energy between molecule and surface is represented by an ab initio six-dimensional potential energy surface. Electron friction coefficients are calculated with density functional theory in a local density approximation. Contrary to previous claims, only minor differences between the adiabatic and nonadiabatic results for dissociative adsorption are found. Our calculations demonstrate the validity of the adiabatic approximation to analyze adsorption dynamics in these two representative systems.
\end{abstract}

DOI: 10.1103/PhysRevLett.100.116102

PACS numbers: 82.65.+r, 34.35.+a, 68.49.Df, 82.20.Kh

The adiabatic Born-Oppenheimer approximation is ubiquitous in the theoretical study of elementary reactive processes at surfaces. Still, there is ample experimental evidence of electronic excitations associated to gas or surface reactions, that can potentially break down the applicability of the adiabatic approach. Electron-hole pairs appear, for instance, in the detection of chemicurrents during the chemisorption of gas-phase species on thin metal films [1,2], as well as in the measurement of electron emission following the scattering of molecules in highlyexcited vibrational states on metal surfaces [3,4]. Although the existence of energy dissipation through electron-hole $(e-h)$ pair excitations is widely accepted, there is not a definite quantitative answer on the role of electronic excitations in the adsorption and reaction rates of diatomic molecules at metal surfaces [5].

Laursen et al. [6] predicted that $e-h$ pair excitations should alter substantially the adsorption dynamics of $\mathrm{H}_{2}$ in $\mathrm{Cu}$ (110). Contravening this prediction, full sixdimensional (6D) adiabatic calculations of the dynamics based on an $a b$ initio potential energy surface have been shown to provide a good description of the experimental results on this system [7]. Measurements of the dissociative adsorption and diffractive scattering of $\mathrm{H}_{2}$ on $\mathrm{Pt}(111)$ are reasonably well described within the adiabatic approximation as well [8]. For heavier molecules, it was claimed that strong energy dissipation effects due to the excitation of $e-h$ pairs are responsible for the strong disagreement between (adiabatic) theoretical and experimental sticking coefficients for $\mathrm{N}_{2}$ on $\mathrm{Ru}(0001)$ [9,10]. Díaz et al. [11] showed afterwards that when the full dimensionality of the process is taken into account, adiabatic calculations are much closer to experiments. This result suggests that nonadiabatic effects might be smaller than previously predicted. Still, the lack of a theoretical calculation, based on state-of-the-art interaction potentials, that explicitly includes the $e-h$ pair excitation channel and the full dimensionality of the process keeps this controversy open.

In this Letter, we evaluate the contribution of $e-h$ pair excitations to the dissociative adsorption of diatomic molecules on metal surfaces. The coupling of low-energy electron excitations can be effectively introduced in the classical equations of motion through a friction coefficient $\eta$ that accounts for energy dissipation [12-15]. The friction picture has been already applied to study the vibrational state of atoms and molecules adsorbed at surfaces [16-18] and the adsorption process itself [19]. However, the complexity of the approach that is required for the accurate description of $\eta$ given in these studies, imposes limitations to comprise the full six dimensionality of the process in the calculation of the dynamics. Our challenge here is to describe the effect of electronic excitations, keeping the six dimensionality of the problem in the whole calculation: the potential energy surface (PES), the friction force, and the dynamics ruled by these forces. To quantify the effect of electron excitations we focus into two representative systems: the dissociative adsorption of $\mathrm{H}_{2}$ on $\mathrm{Cu}(110)$ and that of $\mathrm{N}_{2}$ on $\mathrm{W}(110)$. In $\mathrm{H}_{2} / \mathrm{Cu}(110)$, the dissociation is ruled by a late activation barrier, at short distances from the surface [7]. It is at these distances where the molecule finds high electron densities and where larger energy losses are more likely to occur. In $\mathrm{N}_{2} / \mathrm{W}(110)$, the dynamics is more involved and combines direct and trapping mediated dissociation mechanisms [20,21]. The dissociation of $\mathrm{N}_{2}$ on metal surfaces has been considered as an emblematic example of a system where electronic friction can be relevant. The reasoning behind that assumption is the high value of the friction coefficient for $\mathrm{N}$ atoms moving in electronic media, as will be shown later. We find that the dissociative dynamics in these two represen- 
tative systems, when $e-h$ pair excitations are explicitly included, scarcely differs from the predictions given by the adiabatic calculation.

The molecule-surface interaction is described, within the frozen surface approximation, with an accurate adiabatic 6D PES, built from density functional theory (DFT) calculations $[7,20,21]$. The $6 \mathrm{D}$ PES is interpolated through the corrugation reducing procedure [22]. Nonadiabaticity is introduced in the classical equations of motion through a dissipative force that accounts for electron excitations. Hence, $e-h$ recombination processes are disregarded. For each of the atoms constituents of the molecule, we consider a separate friction force, proportional to the atom velocity, with a friction coefficient $\eta\left(\mathbf{r}_{i}\right)$, where $\mathbf{r}_{i}$ is the vector position of atom $i$. Correlation effects in $\eta$ between the two centers of the molecule have been shown to be minor corrections to the atomic values [23]. The classical equations of motion for each atom of the molecule read

$$
m_{i} \frac{d^{2} \mathbf{r}_{\mathbf{i}}}{d t^{2}}=-\nabla_{i} V\left(\mathbf{r}_{i}, \mathbf{r}_{j}\right)-\eta\left(\mathbf{r}_{i}\right) \frac{d \mathbf{r}_{i}}{d t},
$$

where the first term in the right-hand side is the adiabatic force obtained from the 6D PES $V\left(\mathbf{r}_{\mathbf{i}}, \mathbf{r}_{\mathbf{j}}\right)$ and the second term is the dissipative force.

We base our calculation of $\eta$ on the same grounds of that proposed in Ref. [17]: The motion of the incident particle can be described as a perturbation of the ground state of the coupled molecule or surface system, linear in velocity. As a further approximation, the friction coefficient applied to each atom of the molecule is that of the same atom moving in a homogeneous free electron gas (FEG), with electron density equal to that of the surface at the point in which the atom is placed (local density friction approximation, LDFA). The pioneering work of Refs. [16,17] already showed that this is a good approximation to $\eta$.

In the low-velocity regime, the friction coefficient of an atom inside a homogeneous FEG can be obtained from the scattering properties of the Kohn-Sham wave functions in a static DFT calculation of the coupled system [24]:

$$
\eta=\frac{4 \pi n_{0}}{k_{F}} \sum_{l=0}^{\infty}(l+1) \sin ^{2}\left[\delta_{l}\left(k_{F}\right)-\delta_{l+1}\left(k_{F}\right)\right],
$$

where $n_{0}$ is the FEG density, $k_{F}$ is the Fermi momentum and $\delta_{l}\left(k_{F}\right)$ are the scattering phase shifts at the Fermi level. The friction coefficient so calculated has been widely and successfully used in the context of the stopping power of atoms and ions in the bulk [24,25] and surface regions [26]. For $\mathrm{H}$ atoms penetrating several layers of $\mathrm{Al}$, the TDDFT calculation of Ref. [27] shows that the energy dissipated in the process is very similar to that obtained with the static DFT calculated friction coefficient.

The friction coefficients $\eta$ for $\mathrm{H}$ and $\mathrm{N}$ are shown in Fig. 1 as a function of the mean electron radius of the FEG, $r_{s}=\left[3 /\left(4 \pi n_{0}\right)\right]^{1 / 3}$. We show in the insets the $r_{s}$-values of
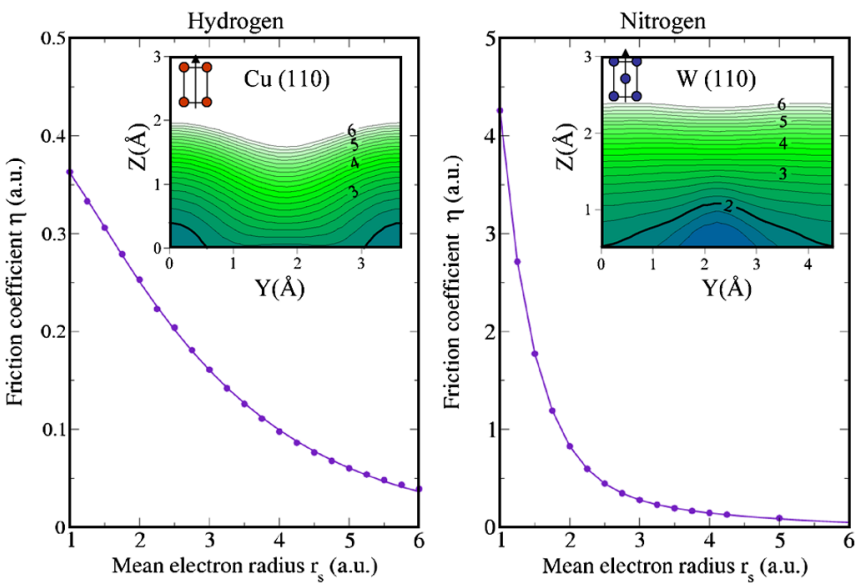

FIG. 1 (color online). Friction coefficient $\eta\left(r_{s}\right)$ in atomic units for: (a) H and (b) N. Values calculated with Eq. (2) are represented by circles. Solid lines show the curve fitting. Insets show the values of $r_{s}$ for $\mathrm{Cu}(110)$ and $\mathrm{W}(110)$ in a plane normal to the surface along the direction represented in each unit cell with an arrow.

$\mathrm{Cu}(110)$ and $\mathrm{W}(110)$ in a plane normal to the surface. The 2D cuts correspond to the electron densities calculated with DFT. The $\eta$-values obtained with the LDFA are of the same order of magnitude of those of Ref. [10]. A direct comparison, however, is difficult since we use a $(6 \times 6)$ friction tensor (diagonal in Cartesian coordinates), instead of the $(2 \times 2)$ tensor given in that reference.

We apply our theoretical approach to analyze the role of electron excitations in the dissociative dynamics of $\mathrm{H}_{2} / \mathrm{Cu}(110)$ and $\mathrm{N}_{2} / \mathrm{W}(110)$. The general methodology is the one of Refs. [7,20,21], respectively: We perform $6 \mathrm{D}$ classical molecular dynamics calculations of the trajectories with and without including electronic friction to calculate the dissociative sticking probability $S_{0}$. The reactive probabilities are derived from a minimum of 5000 trajectories using a conventional Monte Carlo sampling of all possible initial conditions. The statistical samples are identical for the calculations with and without friction. For $\mathrm{H}_{2} / \mathrm{Cu}(110)$ we perform a quasiclassical calculation to account for the initial zero point energy.

The dissociative dynamics of $\mathrm{H}_{2}$ on $\mathrm{Cu}$ is a classical example of activated dissociation at surfaces $[7,28]$. The adiabatic calculation of Ref. [7] for $\mathrm{H}_{2} / \mathrm{Cu}(110)$ shows the existence of a minimum barrier determining the reaction threshold of $592 \mathrm{meV}$, at a distance $Z=0.86 \AA$ from the surface. The calculated $S_{0}$ is shown in Fig. 2 as a function of the incidence kinetic energy $E_{i}$, for different incidence angles $\Theta_{i}$. In all cases, we observe a reduction of $S_{0}$ when electronic friction is taken into account because the inclusion of energy loss channels prevents some of the molecules to overcome the activation barriers. The change obtained is however small in absolute terms. For instance, at normal incidence and for the energy range where friction effects are more clearly visible $(0.75-1 \mathrm{eV})$, most of the 


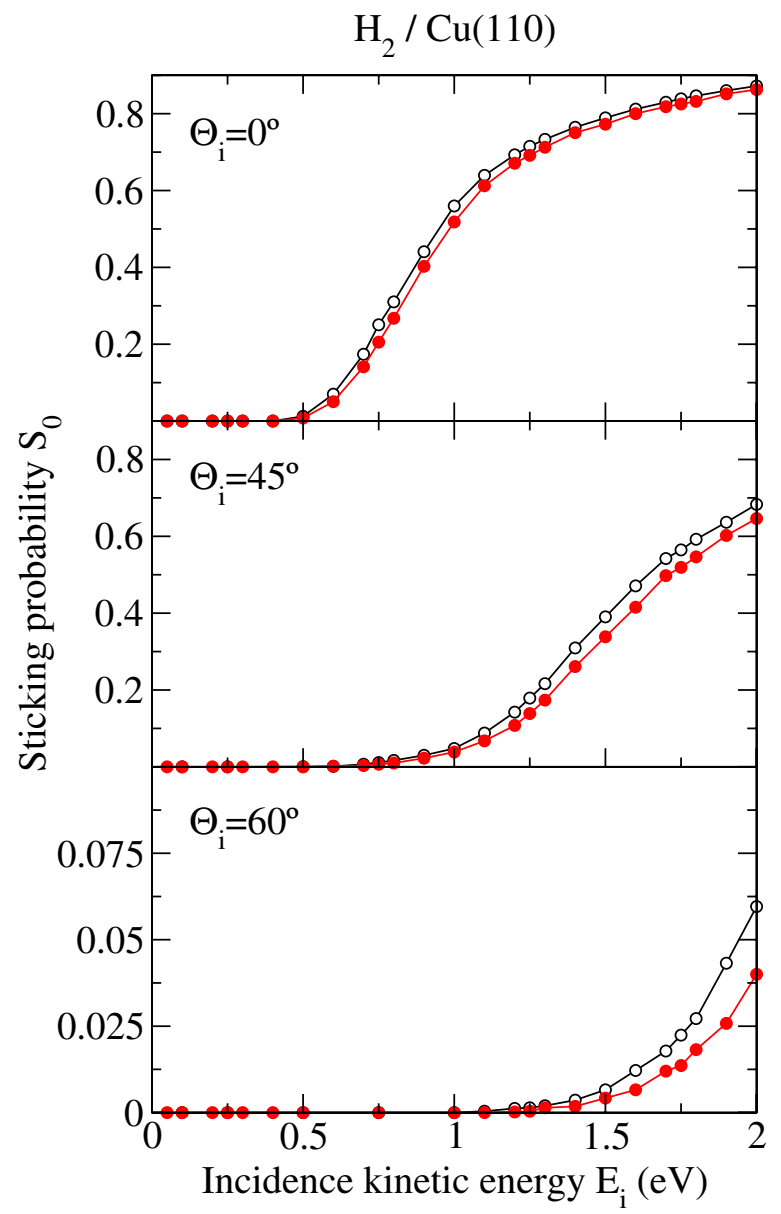

FIG. 2 (color online). Dissociative sticking probability $S_{0}\left(E_{i}\right)$, for $\mathrm{H}_{2} / \mathrm{Cu}(110)$ at different incident angles $\Theta_{i}$. Full red (open black) circles are the results with (without) electronic friction. The statistical error in the calculations is of the order of the size of the circles.

molecules lose less than $50 \mathrm{meV}$ when arriving at distances of $0.86-1 \AA$ from the surface. These energy losses are insufficient to reduce substantially the number of molecules overcoming the barrier to dissociation.

Adiabatic calculations of the dissociative dynamics of $\mathrm{N}_{2}$ on the $\mathrm{W}(110)$ surface $[20,21]$ show that the dynamics depends strongly on $\Theta_{i}$ and $E_{i}$. Close to normal incidence and for energies below $400 \mathrm{meV}$, the dissociative dynamics is characterized by the dynamic trapping of the molecules in the vicinity of the molecular potential well. At higher incident energies or large incidence angles $\left(\Theta_{i}>45^{\circ}\right)$, however, the dissociative reaction is a rather direct process. Figure 3 shows that, when electronic friction is included, there is an increase of $S_{0}$ in most of the cases. The analysis in this system is more complicated due to the strong corrugation of the PES and the resulting complex dynamics. A small number of molecules that show a few rebounds and, as a consequence, spend a comparatively longer time in the region close to the surface, change completely their final stage when friction is included, i.e., from reflected to

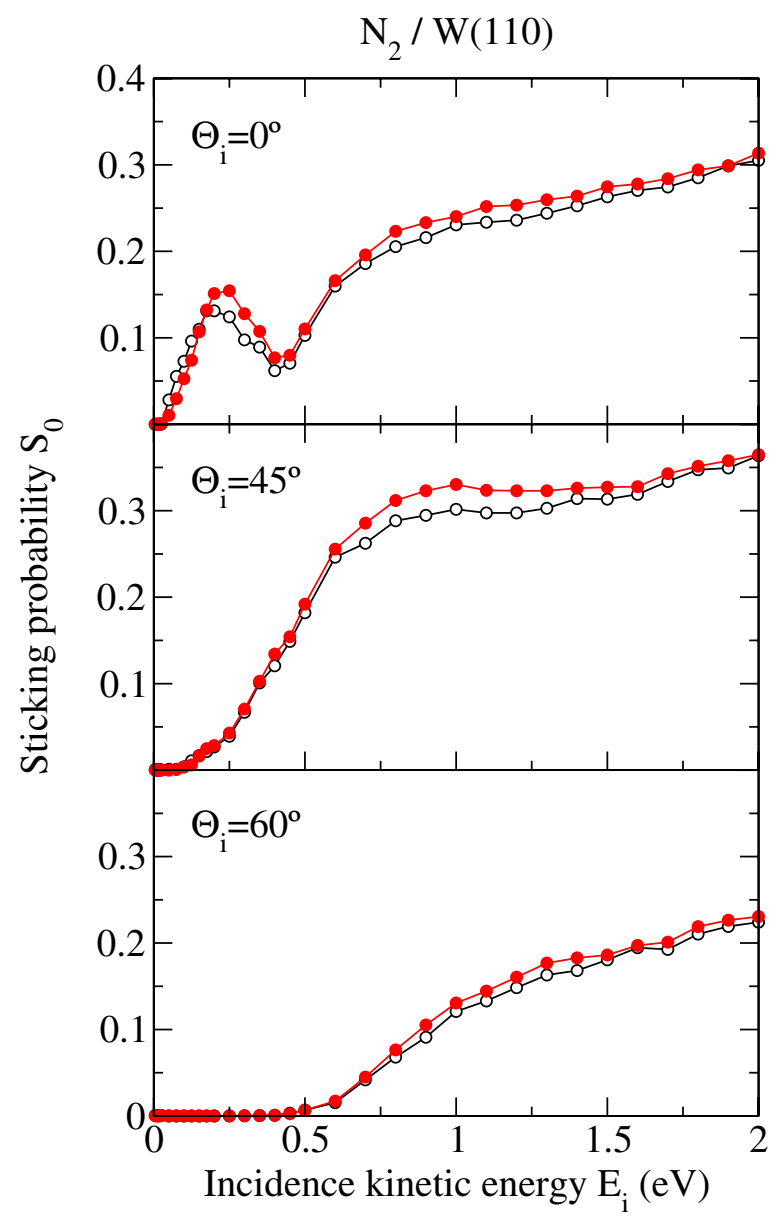

FIG. 3 (color online). Same as Fig. 2 for $\mathrm{N}_{2}$ on W(110).

dissociated and vice versa. The final increase of the nonadiabatic $S_{0}$ shows that, in this region close to the surface, paths to dissociation are in average energetically more favorable than those to reflection.

The important conclusion of our calculations is that for any $\Theta_{i}$ and $E_{i}$, the effect of electronic friction in the dissociative dynamics of these two representative systems is very minor. Such a result shows that the adiabatic calculations represent a valid approximation to study different dissociative dynamics. There is an ultimate reason to understand the marginal role of electronic excitation in the reactive dynamics. We note that in both systems the molecules do probe regions of high electron density where the friction coefficients take their largest values. Nevertheless, this is not enough to infer an energy loss that will affect the dynamics in a significant way. The dissipative force is proportional to both the friction coefficient and the projectile velocity. In the region where the density is high, the molecule-surface potential is highly repulsive. As a consequence, the kinetic energy of the molecules is substantially reduced. This is what makes the dissipative force small, despite the large values of the friction coefficient, and what makes, ultimately, the energy loss a marginal effect. 


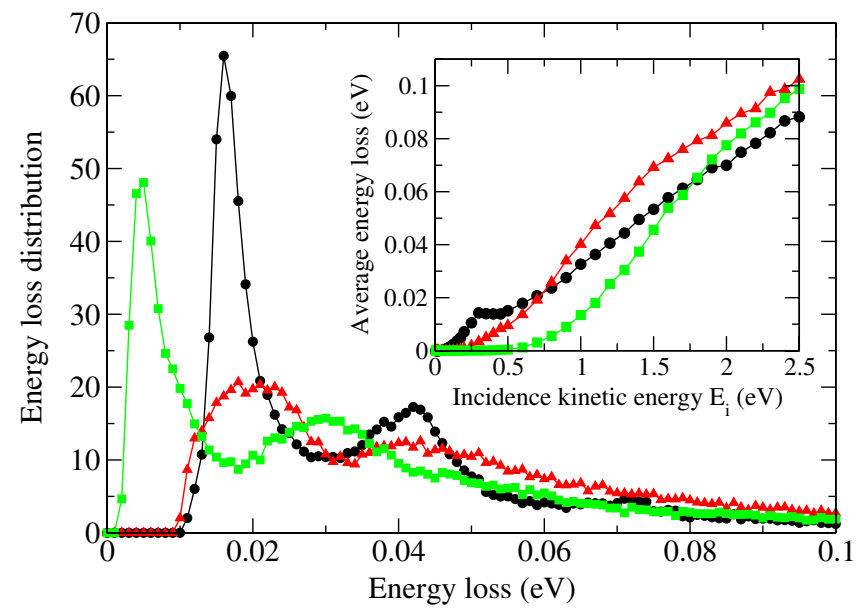

FIG. 4 (color online). Energy loss spectra of the $\mathrm{N}_{2}$ reflected from $\mathrm{W}(110)$ for incidence energy $E_{i}=1.5 \mathrm{eV}$ and incidence angles: $\Theta_{i}=0^{\circ}$ (black circles), $\Theta_{i}=45^{\circ}$ (red triangles), and $\Theta_{i}=60^{\circ}$ (green squares). The average energy loss for each $\Theta_{i}$ and $E_{i}$ is plotted in the inset.

The energy loss spectra of reflected molecules provide direct and quantitative information on the minor role of electron excitations in the scattering process. In Fig. 4 we show the energy loss distribution of $\mathrm{N}_{2}$ with $E_{i}=1.5 \mathrm{eV}$ reflected after scattering from $\mathrm{W}(110)$. The peak structure is related to the regions of closest approach to the surface probed by the reflected molecules. As a general trend, lowenergy loss peaks correspond to molecules reflected far from the surface. Phonon excitations and surface temperature, not included in our approach, will modify the spectra. However, the information it provides is still meaningful: Energy losses due to $e$ - $h$ pair excitations are always below $100 \mathrm{meV}$. Larger measured energy losses should be attributed to a different dissipative channel. Additionally, the inset of Fig. 4 shows that the average energy loss constitutes a few percent of the incident energy.

In summary, we have presented a theoretical approach fully based on DFT calculations to incorporate electronic friction in the $6 \mathrm{D}$ dynamics of molecules interacting with metal surfaces. The role of nonadiabaticity in the dissociative dynamics of $\mathrm{H}_{2} / \mathrm{Cu}$ (110) and $\mathrm{N}_{2} / \mathrm{W}$ (110) is quantitatively determined. Our results show that the contribution of electron excitations is a marginal correction and, therefore, that an adiabatic calculation is still meaningful for a wide range of situations. The low velocity of the reacting molecules in the surface regions of high electronic density is the main reason to explain it. We have also quantified the energy loss spectra of the reflected molecules. For all the incident conditions considered, the energy losses due to electron excitations are always below $100 \mathrm{meV}$. Finally, let us remark that our analysis refers to the case in which $e-h$ pair excitations can be modeled by a friction force; i.e., a continuum of unoccupied states with negligible excitation energy is available. Open questions still remain about the role of $e-h$ pair excitations in other situations, in which nonadiabatic effects are due to the crossing of two or more potential energy curves, with possible transfer of charge included [3,4].

We acknowledge partial support by the Spanish MCyT (Grant No. FIS2007-066711-CO2-00). Computational resources were provided by the SGI/IZO-SGIker at the UPV/ EHU.

[1] B. Gergen et al., Science 294, 2521 (2001).

[2] D. Krix, R. Nünthel, and H. Nienhaus, Phys. Rev. B 75, 073410 (2007).

[3] Y. Huang et al., Science 290, 111 (2000).

[4] J. D. White et al., Nature (London) 433, 503 (2005).

[5] For a review on the dissociation of diatomic molecules at metal surfaces see A. Gross, in The Chemical Physics of Solid Surfaces edited by D.P. Woodruff (Elsevier, Amsterdam, 2003), Vol. 11, Chap. 1.

[6] C. M. Laursen, E. Christoffersen, and G. D. Billing, Chem. Phys. Lett. 290, 275 (1998).

[7] A. Salin, J. Chem. Phys. 124, 104704 (2006).

[8] P. Nieto et al., Science 312, 86 (2006).

[9] L. Diekhöner et al., J. Chem. Phys. 117, 5018 (2002).

[10] A. C. Luntz and M. Persson, J. Chem. Phys. 123, 074704 (2005).

[11] C. Díaz et al., Phys. Rev. Lett. 96, 096102 (2006).

[12] E. G. d'Agliano et al., Phys. Rev. B 11, 2122 (1975).

[13] M. Head-Gordon and J.C. Tully, J. Chem. Phys. 103, 10137 (1995).

[14] J. R. Trail, M. C. Graham, and D. M. Bird, Comput. Phys. Commun. 137, 163 (2001).

[15] J. R. Trail et al., J. Chem. Phys. 119, 4539 (2003).

[16] M. Persson and B. Hellsing, Phys. Rev. Lett. 49, 662 (1982).

[17] B. Hellsing and M. Persson, Phys. Scr. 29, 360 (1984).

[18] V. Krishna and J.C. Tully, J. Chem. Phys. 125, 054706 (2006).

[19] J. T. Kindt et al., J. Chem. Phys. 109, 3629 (1998).

[20] M. Alducin et al., Phys. Rev. Lett. 97, 056102 (2006).

[21] M. Alducin et al., J. Chem. Phys. 125, 144705 (2006).

[22] H. F. Busnengo, A. Salin, and W. Dong, J. Chem. Phys. 112, 7641 (2000).

[23] R. Díez Muiño and A. Salin, Phys. Rev. B 62, 5207 (2000).

[24] P. M. Echenique, R. M. Nieminen, and R. H. Ritchie, Solid State Commun. 37, 779 (1981).

[25] P. M. Echenique et al., Phys. Rev. A 33, 897 (1986).

[26] J. I. Juaristi et al., Phys. Rev. Lett. 82, 1048 (1999).

[27] M. Lindenblatt et al., Nucl. Instrum. Methods Phys. Res., Sect. B 246, 333 (2006).

[28] R. van Harrevelt and U. Manthe, J. Chem. Phys. 123, 124706 (2005). 